Revue internationale de l'économie sociale

Recma

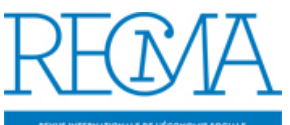

\title{
Le développement européen des sociétés d'assurance mutuelle françaises
}

\section{Tiffany Dumas}

Numéro 325, juillet 2012

ESS : de l'approche entrepreneuriale à une perspective institutionnaliste

The social and solidarity economy: From the entrepreneurial

approach to an instutitionalist perspective

URI : https://id.erudit.org/iderudit/1017425ar

DOI : https://doi.org/10.7202/1017425ar

Aller au sommaire du numéro

Éditeur(s)

Association Recma

\section{ISSN}

1626-1682 (imprimé)

2261-2599 (numérique)

Découvrir la revue

Citer cet article

Dumas, T. (2012). Le développement européen des sociétés d’assurance mutuelle françaises. Revue internationale de l'économie sociale, (325), 111-117.

https://doi.org/10.7202/1017425ar d'utilisation que vous pouvez consulter en ligne. 


\section{LE DÉVELOPPEMENT EUROPÉEN DES SOCIÉTÉS D'ASSURANCE MUTUELLE FRANÇAISES}

par Tiffany Dumas*

* Juriste, doctorante. Mél.: tiffany. dumas@live.com.

(1) Jean Azéma, Groupama, président de la FFSAM, L'Argus de l'assurance, 2007.
(2) Sur ces questions, lire d'Olivier Boned: "Est-il légitime de parler d'identité mutualiste en Europe? Une réponse fondée sur quelques mutuelles de santé", Recma, $n^{\circ} 299$, 2006; "La mondialisation de l'économie induitelle un gommage de l'identité mutualiste? ", Recma, $n^{\circ} 315,2010$; "Gouvernance et contrôle interne à l'aune de Solvabilité II: les nouvelles responsabilités des administrateurs de mutuelles françaises ", Recma, $n^{\circ} 312,2009$ (NDLR).

(3) Aujourd'hui leader mondial de l'assurance des risques industriels, Manufacturers Mutual Fire Insurance Company a été créée en 1835, en Nouvelle Angleterre, pour couvrir les risques $d^{\prime}$ incendie dans les industries textiles (NDLR).

4) Rapport en ligne sur www. recma.org/node/1403 (NDLR).

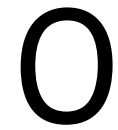
$n$ voit bien que demain nous n'aurons plus une somme de marchés [nationaux], mais un marché européen de l'assurance ${ }^{(1)}$. "La majorité des grands assureurs mutualistes français et européens partagent cette analyse. Les mutuelles ou sociétés d'assurance mutuelle (SAM) souhaitent jouer un rôle significatif dans le marché de l'assurance de demain. Favoriser le rayonnement des assureurs mutualistes sur le plan européen et mondial, faire valoir les spécificités mutualistes et coopératives auprès des pouvoirs publics, mieux répondre aux nouvelles exigences de solvabilité sont désormais leurs enjeux ${ }^{(2)}$. Cependant, les moyens mis à leur disposition ne sont souvent que provisoires et mal adaptés à l'originalité de leur forme juridique. Afin de garantir le succès du mode d'implantation choisi et à défaut de pouvoir se regrouper efficacement au niveau international, les SAM collaborent avec d'autres assureurs.

Ces enjeux et opportunités ne sont pas uniquement ceux des SAM généralistes. Les SAM très spécialisées, comme FM Global (3), ont aussi des possibilités de prendre des positions significatives à l'international. Le risque à terme, dans leur marché domestique de niche, est d'être vulnérable le jour où les grands acteurs décideront de s'y attaquer ou lorsque le nombre de cibles dans cette niche décroîtra pour se concentrer au niveau européen. Depuis 1992, l'émergence d'un marché unique des assurances est, pour l'Union européenne, un objectif central et prioritaire dans la modernisation et l'unification des marchés financiers. Dès octobre 2010, la Commission européenne (CE) a fait des propositions pour donner une place prépondérante aux SAM dans le marché unique des assurances. Le 10 mars 2011, le Parlement européen s'est engagé à missionner la CE afin que soit lancée "l'étude sur la situation des mutuelles dans tous les Etats membres pour examiner notamment leurs activités transfrontières "; un rapport lui a été remis en juillet $2011^{(4)}$. Selon les institutions politiques et la profession, les SAM sont un vecteur essentiel pour promouvoir une Europe plus démocratique et sociale. Elles correspondent exactement aux valeurs que l'Union européenne veut véhiculer et qui sont définies dans les objectifs du traité de Lisbonne (préambule et article 3.3).

Les entraves législatives n'ont pourtant pas empêché les SAM françaises, petites ou grandes, de s'implanter au-delà des frontières nationales. 


\section{Un régime d'implantation taillé pour les sociétés de capitaux}

Le droit communautaire a dessiné trois régimes d'implantation à l'étranger, fondés sur les libertés de prestation de services et d'établissement. Il prend pour modèle la société par actions, bien que ces régimes soient difficilement adaptables aux SAM désireuses de maintenir leur originalité.

\section{Régime de libre prestation de services (LPS)}

Le régime d'implantation des sociétés d'assurance mutuelle le plus répandu est celui de la libre prestation de services. Elles existent sous cette forme dans la majorité des Etats de l'Espace économique européen, y compris en Pologne, où l'on ne retrouve qu'une mutuelle nationalisée.

Ce régime strict est utilisé par les SAM dans les activités assurantielles qu'elles distinguent de leurs activités de distribution. Ces dernières ne sont plus sous le contrôle direct de la SAM et font l'objet d'accords de distribution avec des assureurs partenaires étrangers. Elles sont aussi fréquemment déléguées à une joint-venture locale ou proposées à des courtiers. La prudence impose de séparer ces activités pour ne pas risquer de les voir requalifiées sous le régime de libre établissement. En effet, la frontière entre ces deux régimes est mince. La difficulté se trouve notamment dans le lien que les SAM entretiennent avec leur réseau de distribution.

L'implantation sous le régime de la libre prestation de services constitue ainsi une solution efficace pour s'implanter rapidement, avec une structure simple et peu coûteuse. La SAM peut obtenir l'agrément pour s'établir dans un nouvel Etat membre sous un mois et reste soumise à l'autorité de contrôle et à la législation d'origine sans effectuer de modification statutaire.

Cependant, ce régime n'est pas destiné à une implantation permanente, puisque le montant des cotisations perçues sur trois années est soumis à un plafond. En pratique, les SAM sont également contraintes de prévoir un personnel assurantiel formé aux conflits de réglementation, voire linguistiques, entre l'Etat d'origine et l'Etat d'accueil. Par la scission de son activité de distribution, elle n'est pas maîtresse de sa stratégie commerciale, ce qui limite aussi ses perspectives de croissance.

\section{Régime de libre établissement}

(5) « Comment sortir de la crise par une intégration transfrontalière des entreprises de l'économie sociale ", intervention de Luc Roger, directeur des études européennes et internationales d'Harmonie Mutuelles, le 22 octobre 2009 au Conseil économique et social européen, lors du séminaire "Sortir du tunnel: créer un environnement favorable à une économie sociale " (www. eesc.europa.eu).
Le régime de libre établissement est plus contraignant que celui de libre prestation de services. Il oblige l'entreprise à créer une succursale et à tenir une double comptabilité, la fiscalité relevant en partie du droit local et de l'Etat d'origine. Harmonie Mutuelles est la seule SAM à avoir opté pour une implantation sous le régime d'établissement, même si ce choix ne semblait pas être celui originellement prévu ${ }^{(5)}$. En attendant la mise en place juridique d'un statut de mutuelle européenne, elle utilise cette solution transitoire sur trois ans pour son développement international, notamment en Italie. 
(6) Facts \& Figures, "En quoi une mutuelle d'assurance diffère d'une société anonyme ", rapport, version 1.01, 27 juin 2008.
L'avantage et l'inconvénient de la succursale, c'est qu'elle reste sous le régime statutaire de la SAM. La succursale est une branche de la SAM et dépend juridiquement d'elle. Elle répond ainsi au principe de mutualité des fonds. Etablie de manière permanente dans l'Etat membre, la SAM doit ajouter aux obligations locales en matière d'assurance des obligations comptables et fiscales, exonérées sous le régime de LPS. Elle doit donc procéder à une comptabilité très laborieuse pour se conformer aux réglementations de son Etat d'origine et de son Etat d'accueil. Ce régime s'avère donc très complexe et finalement plus contraignant qu'un régime de filiales détenues à $100 \%$ par la société mère. Dans ce cas, l'indépendance de la filiale n'est que formelle, puisqu'elle reste politiquement sous la direction de la société mère.

\section{La filiale}

Le dernier régime d'implantation est celui de la création d'une société par actions à vocation assurantielle, entièrement régie par le droit local étranger et détenue majoritairement par la société mère. Ce dispositif demande une grande libération de fonds, difficile pour de nombreuses structures. Si ce choix d'implantation apparaît comme le plus contradictoire avec la nature même d'une mutuelle, les SAM européennes et françaises y ont recours. Groupama est un des premiers groupes mutualistes à entreprendre un développement à l'international. Son chiffre d'affaires réalisé hors de France est en croissance depuis 2006, atteignant $26,4 \%$ en 2009 avec une implantation dans treize pays.

L'étude de Facts \& Figures ${ }^{(6)}$ le confirme: la méthode d'implantation par la démutualisation, la fusion et les acquisitions est très risquée pour les sociétés mutualistes qui ne désirent pas perdre leur identité. En Espagne, Groupama a formé sa filiale au fur et à mesure de créations, d'acquisitions et de fusions de sociétés françaises déjà implantées (notamment Gan et Azur) et de sociétés espagnoles. Elle ne met d'ailleurs pas en avant des valeurs mutualistes dans sa filiale espagnole. Néanmoins, la filiale peut être une option viable si elle est créée progressivement par la SAM. Cette alternative s'avère plus simple à gérer que la succursale. La filiale est soumise à un seul droit national et reste sous la direction de la société mère, même si celle-ci est d'une autre nationalité.

\section{Quelles structures de coopération internationale?}

Le partenariat est la solution classique pour une alliance ponctuelle. Harmonie Mutuelles a choisi cette modalité avant de se constituer en mutuelle européenne. La majorité des mutuelles voulant se développer ont toutefois préféré créer ou intégrer une structure plus solide, avec ou sans but assurantiel. 


\section{Les structures de coopération communes sans but assurantiel} Le groupement européen d'intérêt économique (GEIE)

Euresa est un groupement unique en son genre. Il associe de nombreuses SAM en Europe, leaders sur leur marché domestique. A travers cette structure, elles mettent en commun leurs moyens sans avoir de direction commune. Ce GEIE s'engage au niveau européen en définissant et en communiquant les valeurs et les engagements rassemblant ses membres, notamment l'économie citoyenne, le développement durable et socialement responsable. Il soutient la coopération, l'échange de savoir-faire et le développement d'entreprises d'assurance liées à l'économie sociale en Europe. La holding permet la création de joint-ventures dans d'autres pays européens. L'institut sert d'outil de réflexion et de formation pour la protection sociale, mais aussi pour les stratégies et les politiques européennes de ses membres. Il s'agit d'une plateforme de coopération entre grands acteurs mutualistes européens, où chacun apporte et reçoit en retour, dans une recherche de rapports entre " égaux ». Ces mutualistes sont des généralistes qui cherchent à atteindre la cible la plus large. L'idée de cette structure est de créer des moyens pour mettre en œuvre le développement international des mutualistes généralistes. Ces derniers veulent ainsi être aussi efficaces que les assureurs capitalistes sans avoir à se démutualiser pour leur financement. Hébergeant le comité de pilotage, principale instance décisionnaire du GEIE, Euresa a clairement pour but de remplir le vide juridique d'une structure mutualiste à l'échelle européenne, qui aurait été le pendant de la société européenne. Néanmoins, un GEIE n'a pas de vocation assurantielle et ne permet pas une solidarité financière entre ses membres.

\section{La société anonyme suisse}

Les intentions d'Eurapco sont très proches de celles d'Euresa, avec cette différence qu'il existe à la tête de la société anonyme une direction en mesure d'imposer les tendances stratégiques aux différents actionnaires. Elle compte des assureurs mixtes (capitalistes et mutualistes) figurant dans les dix premiers de leur marché domestique respectif. Même si beaucoup de ses actionnaires sont ou étaient des mutualistes, l'aspect mutualiste et d'économie sociale est clairement mis en retrait dans leur présentation, contrairement à Euresa. Il s'agit d'une plateforme dynamique, profitant des expertises locales d'autres assureurs à succès en Europe.

\section{La société coopérative européenne}

Harmonie Mutuelles a fondé avec l'assureur italien Cesare Pozzo une société coopérative européenne, détenue par chacun à $50 \%$. Elle est propriétaire des contrats et acteur de la distribution. L'avantage de cette forme est son statut européen et la mise en commun de moyens. Toutefois, le renvoi continuel aux législations nationales divergentes rend sa gestion compliquée et finalement peu utilisée. Plus qu'un simple partenariat, cette alliance doit consolider les liens entre les deux mutuelles, qui veulent développer leur présence sur le marché collectif transalpin de la complémentaire 
santé. Or, cette structure n'a pas la vocation d'assurer, d'où l'implantation parallèle d'Harmonie Mutuelles sous forme de succursale en Italie. Elle compte ainsi atteindre de nouveaux marchés de la complémentaire santé pour se détacher du marché français saturé. Harmonie Mutuelles espère pouvoir prochainement regrouper sa société coopérative européenne et sa succursale en une seule mutuelle européenne.

\section{Les alliances avec un but assurantiel}

\section{Les joint-ventures}

Au lieu de créer une filiale supportée par une seule entreprise, la jointventure est partagée entre plusieurs assureurs, réduisant ainsi les coûts de création et de gestion. Principalement destinée aux activités de distribution, elle est un instrument pour faire connaître les marques mères à travers une société locale. Cependant, ce partage de pouvoirs suppose une bonne entente entre les partenaires. L'unique forme efficace est la société par actions. Plus rien n'oblige les partenaires à garder leurs valeurs mutualistes dans la société et à ne pas être tentés par la cotation en Bourse.

\section{Les rachats}

L'acheteur acquiert immédiatement les compétences-métiers et un réseau de distribution locaux confirmés. C'est, avec la fusion, le mode classique d'implantation des sociétés par actions, mis en œuvre par Groupama par exemple. Or, ces modes s'avèrent très risqués pour les mutuelles. L'effort financier est important. Pour effectuer ces opérations de croissance externe, certaines mutuelles se démutualisent par manque de fonds. Le croisement de cultures mène à des difficultés dans le management des sociétés acquises, débouchant sur des problèmes de solvabilité. De nombreux rachats ont ainsi échoué (Norwich Union absorbé par Aviva ou Trygg-Hansa absorbé par Royal \& Sun Alliance). Certaines mutuelles, plus prudentes, préfèrent ainsi ne prendre qu'une participation dans le capital, comme la Maif dans Unipol (Italie) et Vivium (Belgique) ou MMA et Maaf dans le capital de Caser Seguros (Espagne). Cette solution n'implique pas l'obligation de gérer les sociétés et se traduit par une responsabilité limitée en matière de solvabilité.

\section{De la SGAM au statut de mutuelle européenne?}

La société de groupe d'assurance mutuelle (SGAM) constitue une bonne piste pour une implantation efficace des SAM. Cependant, la confrontation d'ordres juridiques différents complique son application, ce que permettrait d'éviter un statut européen de la mutuelle.

\section{La société de groupe d'assurance mutuelle (SGAM)}

La SGAM est une originalité française datant de 2001. Il s'agit d'une fusion de fait ou d'une opération de concentration, conformément 
(7) Depuis 2003, Covéa regroupe la Maaf, MMA et la GMF. Fin 2009, la Macif, la Maif et la Matmut s'unissaient au sein de Sferen (NDLR).

(8) "Actualités ", Recma, $n^{\circ} 323$ (NDLR).

(9) Lire "Actualités" dans ce numéro (NDLR). à l'article L 430-1 du Code du commerce. Une structure mère est créée pour gérer la mise en commun de moyens financiers, assurantiels et humains et pour imposer les grandes lignes stratégiques des SAM filiales. Elle permet aux SAM françaises de réunir leurs forces et d'être financièrement solidaires comme leurs concurrentes capitalistes, tout en gardant leur forme mutuelle. Ce statut s'avère efficace et satisfaisant au niveau national si l'on considère le nombre de SGAM en France ${ }^{(7)}$. Covéa et l'assureur belge Ethias ont même tenté cette voie pour se rapprocher. Cependant, en utilisant un droit national, il n'existe pas de reconnaissance au niveau européen, ce qui constitue un frein au développement à l'international. De plus, les divergences entre les droits nationaux compliquent la gestion pour la SAM étrangère: membre d'une SGAM, elle perd indirectement sa nationalité, puisqu'elle est dirigée par une superstructure française.

\section{Le statut de mutuelle européenne}

Les groupes mutualistes français (Euresa, Macif, MGEN, MAE, Maif, Groupama, Harmonie Mutuelles...) et d'autres nationalités militent auprès des institutions européennes pour la création d'un statut de mutuelle européenne ${ }^{(8)}$. Ils veulent promouvoir la création d'une forme proche de celle de la SGAM, afin d'avoir une réponse mutualiste au mouvement de consolidation des sociétés d'assurance. Ils pensent ainsi former des grands groupes comparables à ceux des sociétés par actions multinationales pour mieux résister à la concurrence accrue du marché de l'assurance ${ }^{(9)}$.

Il s'agit, en d'autres termes, de réunir en une seule superstructure toutes les implantations individuelles des assureurs-mutuelles et leurs structures de soutien formées avec leurs partenaires. Cette superstructure doit avoir un régime juridique unifié, tout en respectant les particularités nationales et mutualistes de ses membres. Cette mutuelle européenne aura ainsi parfaitement la possibilité de répondre aux nouvelles exigences de solvabilité et de concurrencer les groupes d'assureurs capitalistes multinationaux. Grâce à la solidarité financière des membres, elle doit permettre aux mutuelles de mieux se développer sans passer par la démutualisation ou la cotation en Bourse. L'intégration dans une telle structure implique, en conséquence, un abandon certain de l'indépendance et de la souveraineté de la société mutuelle, si les modalités de sortie sont aussi rigides que pour les SGAM.

\section{Conclusion}

Principalement installées sous le régime de la libre prestation de services, les implantations à l'étranger de mutuelles et de sociétés d'assurance mutuelle sont confondues dans l'entité principale. Ces entreprises prennent rarement l'initiative de publier les informations sur la taille 
du portefeuille et les résultats dégagés à l'étranger : une mutuelle considère ses adhérents français et étrangers comme un tout.

Disposer des chiffres des joint-ventures est tout aussi compliqué, puisqu'elles sont juridiquement indépendantes des SAM et regroupent plusieurs assureurs de différents pays. Les joint-ventures des SAM sont des sociétés assez discrètes et les informations les concernant restent très rudimentaires. Les SAM qui détiennent une joint-venture l'utilisent comme un instrument, un soutien, et préfèrent mettre en avant leur propre marque. Elles restent en général discrètes sur leur jointventure, au statut contradictoire avec les valeurs mutualistes qu'elles promeuvent au niveau européen. Cette discrétion s'explique aussi par la volonté des SAM d'intégrer ces structures dans une entité commune européenne, idéalement sous le statut de mutuelle européenne.

A défaut d'un modèle européen plus respectueux de leurs spécificités, les mutuelles d'assurance utilisent pour l'heure les dispositifs juridiques pensés pour les sociétés de capitaux, unique modèle, jusqu’à présent, pour le législateur européen. 